PSS PROCEEDINGS

\title{
Final state QCD studies at LEP: Part II
}

\author{
Nuno Anjos* ${ }^{\dagger}$ \\ LIP, Portugal \\ E-mail: nuno@lip.pt
}

We review two studies of $\gamma \gamma$ collisions performed by DELPHI at LEP2, the first on single particle inclusive production, the second on di-jet production. We also briefly highlight the comprehensive study of $e^{+} e^{-} \rightarrow q \bar{q}$ events performed by L3 at LEP1 and LEP2.

International Europhysics Conference on High Energy Physics

July 21st - 27th 2005

Lisboa, Portugal

\footnotetext{
${ }^{*}$ Speaker.

${ }^{\dagger}$ I am grateful for the invitation from the organizing committe to participate in this conference and to the support of FCT.
} 


\section{Introduction}

With a precisely measured centre-of-mass energy, a well defined initial state void of QCD activity and no beam remnants, the LEP experiments (ALEPH, DELPHI, L3 and OPAL) were an ideal environment for QCD studies.

The main source of hadron production at LEP2 were two-photon $(\gamma \gamma)$ collisions. Anti-tagged colisions, where both electron and positron escape through the beam pipe and the photons have low virtualities, are of special interest. Large $p_{T}$ processes involving quasi-real photons are sensitive to the quark and gluon components of the resolved photons. The analysis of high $p_{T}$ jet production complements the studies of deep-inelastic scattering of a quasi-real photon, which probes the quark component. Together, they allow the measurement of the parton density function of the photon.

The most interesting sample for QCD studies at LEP were $e^{+} e^{-} \rightarrow q \bar{q}$ events. During the LEP1 phase each experiment recorded about 4 million hadronic events at the $\mathrm{Z}$ pole. This high statistics, combined with very low background, allowed precise measurements of the hadronic event structure and detailed QCD tests and studies. Further, events with an observed high energy photon, which have lower effective centre-of-mass energy due to ISR or FSR, enable studies of the energy dependence of strong interaction at energies bellow the $\mathrm{Z}$ pole. During the LEP2 phase data was taken at centre-of-mass energies from 130 to $209 \mathrm{GeV}$. The statistics at these energies is reduced with respect to the $\mathrm{Z}$ pole because the hadronic cross-section his much lower, ISR brings down the effective centre-of-mass energy of many events to the $Z$ pole, and background (mainly from WW and ZZ fully hadronic decays) is much larger. Nevertheless these events extend the energy range over which QCD can be tested well above the $\mathrm{Z}$ pole.

\section{Single Particle Inclusive Production in $\gamma \gamma$ Collisions with DELPHI at LEP2}

The data used for this analysis [1] were collected by the DELPHI detector at centre-of-mass energies from $161 \mathrm{GeV}$ to $209 \mathrm{GeV}$, corresponding to an integrated luminosity of $617 \mathrm{pb}^{-1}$.

After the selection of charged and neutral tracks, about 910k hadronic two-photon events were extracted through the application of the following selection criteria:

- Low angle anti-tag: energy deposited in the DELPHI luminosity monitor (STIC: $2.5^{\circ}<$ $\left.\theta_{\text {STIC }}<9^{\circ}\right) E_{\text {STIC }}<30 \mathrm{GeV}$;

- Selection of hadronic events: number of charged tracks $N_{c h}>4$;

- Backgroud supression: visible invariant mass $5 \mathrm{GeV} / c^{2}<W_{\text {vis }}<35 \mathrm{GeV} / c^{2}$ (calculated from charged and neutral tracks);

- Improve measurement of $p_{T}$ : acceptence for charged particles limited in pseudorapidity to $|\eta|<1$, or $|\eta|<1.5$.

The $p_{T}$ spectrum of the charged particles of the selected events is presented in Fig. 1(left) with the expected signal and background contributions, obtained from Monte Carlo and normalized to the data integral luminosity. Hadronic $\gamma \gamma$ events were generated with PYTHIA. The $e^{+} e^{-} \rightarrow q \bar{q}$, $e^{+} e^{-} \rightarrow$ four-fermion, $\gamma \gamma \rightarrow \tau^{+} \tau^{-}$and $e^{+} e^{-} \rightarrow \tau^{+} \tau^{-}$backgrounds were generated with PYTHIA, EXALIBUR, BDKRS and KORALZ 4.2, respectively. The data are well reproduced by the simulated events. The differential inclusive charged hadron cross-section distribution $d \sigma / d p_{T}$ was 

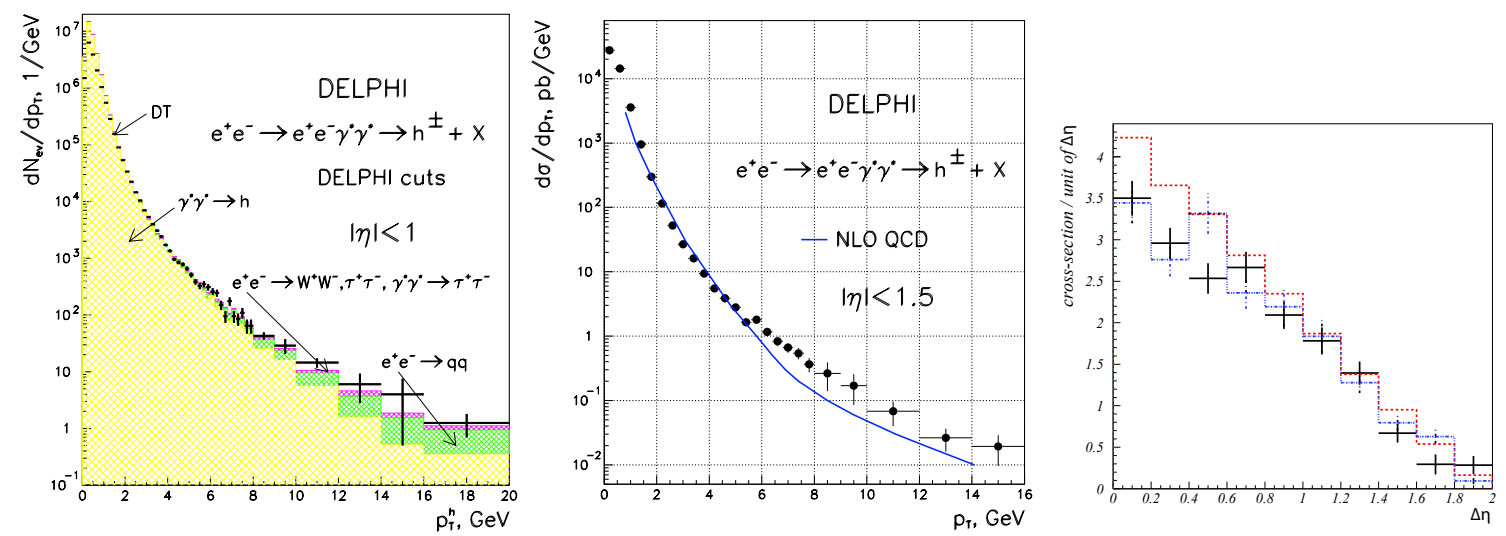

Figure 1: Charged particle $p_{T}$ spectrum (left), charged particle cross-section (centre) and di-jet cross-section compared with LO (dotted) and NLO (dashed) QCD calculations (right) in $\gamma \gamma$ collisions.

extracted from the experimental $d N / d p_{T}$ data and is compared with the NLO QCD prediction in Fig. 1(centre). One sees some excess of data for $p_{T}>6 \mathrm{GeV} / c$ but not at the level of L3 [2]. Below $6 \mathrm{GeV} / c$ the data follow well the NLO QCD prediction, as observed by OPAL [3].

\section{Di-jet Production in $\gamma \gamma$ Collisions with DELPHI at LEP2}

The data used for this analysis [4] were collected by the DELPHI detector at centre-of-mass energies from $189 \mathrm{GeV}$ to $209 \mathrm{GeV}$, corresponding to an integrated luminosity of $550 \mathrm{pb}^{-1}$.

In this analysis jets are reconstructed from charged tracks and photons. After selecting these objects in the detector, 5147 events were selected by the application of a set of criteria similar to the one presented in chapter 2: $N_{c h} \leq 5, W_{v i s}<35 \mathrm{GeV} / c^{2}, E_{\text {STIC }}<25 \mathrm{GeV}$. An additional criteria for background suppression is that the total transverse momentum mut be bellow $30 \mathrm{GeV} / c$. Two jets are reconstructed by the $k_{\perp}$ algorithm implemented in KTCLUS. Each jet must have $|\eta|<1$ and $p_{T}>3 \mathrm{GeV} / c$. The average $p_{T}$ of jets must be above $4 \mathrm{GeV} / c$ to guarantee asymmetry. Soft remnant jets are allowed outside the mentioned $\eta$ domain. Hadronic $\gamma \gamma$ events are simulated with PYTHIA. The $e^{+} e^{-} \rightarrow q \bar{q}$ background was simulated with KK2f and contributes $(500 \pm 5)$ events. The $\gamma \gamma \rightarrow \tau^{+} \tau^{-}$contamination is estimated with BDKRS to be $(43 \pm 3)$ events, while $e^{+} e^{-} \rightarrow \tau^{+} \tau^{-}$the background is negligible. Other backgrounds are four-fermion processes, with $(38 \pm 4)$ events, multiple parton interactions, with $(131 \pm 7)$ events, and $(893 \pm 13)$ non 2 -to-2 jets events. Hadron prduction in collisions of quasi-real photon can occur through direct (Dir), singleresolved (SR) or double-resolved processes (DR), if none, one or both photons are resolved into their hadronic components, respectively. The phase-space domains where each process dominates were studied separately. Simulation does not describe well the 'DR' domain, where contributions from both quark-parton (QPM) and vector-dominance (VDM) models must be considered. A 3-parameter fit of the data on the $E_{\text {out }}$, transverse momentum balance and total invariant mass distributions is performed in each one of the phase-space domains, and is used to correct the simulation. The simulated background is then subtracted from the data. The distributions of the jets $\overline{P_{T}}$ and $|\Delta \eta|$ are then corrected for hadronization effects and detector acceptance using simulation. The differential cross-section is then extracted, and in shown Fig. 1(right) to be in good agreemant with 

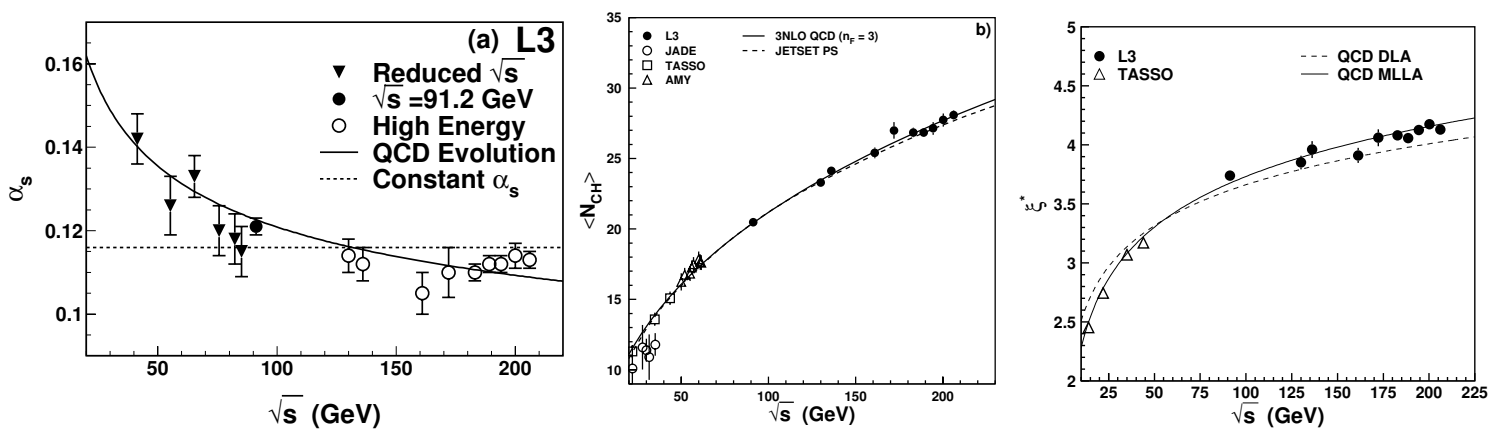

Figure 2: Evolution with energy of $\alpha_{s}$ (left), average charged multiplicity (centre) and $\xi^{*}$ (right).

QCD NLO predictions. The total di-jet cross-section is measured as $(17.1 \pm 0.3) \mathrm{pb}$, to be compared with expectations of $(20.2 \pm 0.1) \mathrm{pb}$ and $(17.8 \pm 0.1) \mathrm{pb}$ for LO and NLO QCD calculations, respectively.

\section{Studies of Hadronic Event Structure in $e^{+} e^{-} \rightarrow q \bar{q}$ from 30 to $209 \mathrm{GeV}$ with $\mathrm{L3}$}

The comprehensive study of hadronic event structure in $e^{+} e^{-} \rightarrow q \bar{q}$ events [5] performed by L3 is too extensive to be reviwed here, thus we just present its main results. The study covers a very large energy range, from 30 to $209 \mathrm{GeV}$. The low energy range is explored using events with isolated high energy photons, while the high energy range is explored using the LEP2 events.

Hadronic event shapes are studied extensively. From this study the strong coupling constant is measured to be $\alpha_{s}\left(m_{Z}\right)=0.1227 \pm 0.0012$ (exp.) \pm 0.0058 (theo.). The measured energy evolution of $\alpha_{s}$ is presented in Fig. 2(left). Effects of soft gluon coherence are studied through charged particle multiplicity and momentum distributions. The evolution with energy of the average charged particle multiplicity is shown in Fig. 2(centre) to be compatible with NNNLO QCD calculations. The evolution with energy of the peak of the $\xi_{p}=\ln \left(1 / x_{p}\right)$ spectrum, $\xi^{*}$, is shown in Fig. 2(right) to be compatible with MLLA QCD calculations.

\section{Summary}

Two studies of $\gamma \gamma$ collisions performed by DELPHI at LEP2, the first on single particle inclusive production [1], the second on di-jet production [4] were reviewed. The comprehensive study of $e^{+} e^{-} \rightarrow q \bar{q}$ events performed by L3 at LEP1 and LEP2 [5] was briefly highlighted.

All results are preliminary

\section{References}

[1] M. Chapkin, V. Obraztsov, A. Sokolov, Ph. Gavillet, DELPHI 2005-018 CONF-738.

[2] P. Achard et al., L3 Collab., CERN-EP/2002-081.

[3] K. Ackerstaff et al., OPAL Collab., Eur. Phys. J. C6 (1999) 253.

[4] V. Pozdnyakov, Yu. Vertogradova, DELPHI-2005 CONF-727.

[5] P. Achard et al., L3 Collab., CERN-PH-EP/2004-024. 